\title{
Digital Transformation in Service and Computing Oriented Manufacturing
}

\author{
Theodor Borangiu, Damien Trentesaux, André Thomas, Paulo Leitão \\ and José Barata Oliveira
}

This volume gathers the peer reviewed papers which were presented at the sixth edition of the International Workshop "Service Orientation in Holonic and Multi-agent Manufacturing-SOHOMA'16" organized on October 6-7, 2016 by the New University of Lisbon, Portugal in collaboration with the CIMR Research Centre in Computer Integrated Manufacturing and Robotics of the University Politehnica of Bucharest, Romania, the LAMIH Laboratory of Industrial and Human Automation Control, Mechanical Engineering and Computer Science of the University of Valenciennes and Hainaut-Cambrésis, France and the CRAN Research Centre for Automatic Control, Nancy of the University of Lorraine, France.

\footnotetext{
T. Borangiu (四)

Department of Automation and Industrial Informatics,

University Politehnica of Bucharest, Bucharest, Romania

e-mail: theodor.borangiu@cimr.pub.ro

D. Trentesaux
}

LAMIH, CNRS UMR 8201, Université de Valenciennes et du

Hainaut-Cambrésis, Lille, France

e-mail: damien.trentesaux@univ-valenciennes.fr
A. Thomas
CRAN, CNRS UMR 7039, Université de Lorraine, Nancy, France
e-mail: andre.thomas@univ-lorraine.fr
P. Leitão
Artificial Intelligence and Computer Science Laboratory,
Polytechnic Institute of Bragança, Porto, Portugal
e-mail: pleitao@ipb.pt
J. Barata Oliveira
Faculdade de Ciências e Tecnologia, Universidade Nova de Lisboa,
Caparica, Portugal
e-mail: jab@uninova.pt 
The main objective of the SOHOMA workshops is to foster innovation in sustainable manufacturing and in this context to promote concepts, methods and solutions addressing trends in the service orientation of agent-based control technologies with distributed intelligence and the integration with enterprise management.

The book is structured in eight parts, each one grouping a number of chapters describing research in actual domains of the digital transformation in manufacturing and trends in future service and computing oriented manufacturing control: Part 1: Cloud and Cyber-Physical Systems for Smart Manufacturing, Part 2: Reconfigurable and Self-organized Multi-Agent Systems for Industry and Service, Part 3: Sustainability Issues in Intelligent Manufacturing Systems, Part 4: Holonic and Multi-agent System Design for Industry and Service, Part 5: Should Intelligent Manufacturing Systems be Dependable and Safe?, Part 6: Service-oriented Management and Control of Manufacturing Systems, Part 7: Engineering and Human Integration in Flexible and Reconfigurable Industrial Systems, Part 8: Virtualization and Simulation in Computing-oriented Industry and Service.

These eight evolution lines have in common concepts, methodologies and implementing frameworks for Digital transformation in service and computing oriented manufacturing.

By defining an Internet-scale platform for networked production encapsulating the right abstractions to link effectively and scalably the various stakeholders of the Manufacturing Value Chain (materials and component producers, manufacturing plants, technology providers, services, and integrators) the actual vision and initiatives about developing generic architectures and core technologies for the Digital Transformation of Manufacturing (DTM) are presented in the research reported in the workshop, and included in the present book.

An effect of this initiative is shifting from good-dominant logic to servicedominant logic (also expressed as Product-Service Extension] which enhances the utility of product delivered to customer, e.g., installing, configuring/tuning, training the customer, repairing, maintaining/upgrading) adding value in customer operations.

The global vision for DTM refers to: (i) Pervasive instrumenting manufacturing resources, materials flows and environments, (ii) Interconnecting orders, products and resources in a secured Industrial Internet of Things, (iii) Taking smart decisions in production management and control by distributing intelligence among multi-agent systems (MAS) acting as information counterparts of physical assets, and orchestrating production workflows as manufacturing services in Service Oriented Architectures (SOA).

New developments are related to the digital transformation of manufacturing; they are described in this book.

Cloud manufacturing (CMfg), one of these new lines, has the potential to move from production-oriented manufacturing processes to customer- and service-oriented manufacturing process networks, e.g. by modelling single manufacturing assets as services in a similar way as SaaS or PaaS software service solutions. In CMfg all manufacturing resources and abilities for the manufacturing 
life cycle can be provided in different service models. The Industrial IoT (IIoT) integrated in the cloud allows creating novel network architectures seamlessly integrating smart connected objects, and distinct cloud service providers. The IIoT represents a core enabler for product-centric control and increasing servitization: the product directly requests processing, assembly and materials handling from available providers while it is in execution and delivery. The product monitors its own status; notifies the user when something goes wrong; helps the user to find and access the necessary product-related models and information from manufacturers in the CMfg ecosystem, and eases the synchronization of product-related data and models.

To achieve high levels of productivity growth and agility to market changes, manufacturers will need to leverage Big Data sets to drive efficiency across the networked enterprise. A number of contributions formulate proposals for a framework allowing the development of Manufacturing Cyber Physical Systems (MCPS)_ICT systems (sensing, actuating, computing, communication) embedded in physical objects, interconnected through several networks including the Internet, and providing businesses with a wide range of innovative applications based on digitalized data, information and services. MCPS include capabilities for complex event processing and Big Data analytics, which are expected to move the manufacturing domain closer towards digital- and cloud manufacturing within the Contextual Enterprise.

Projects are described related to the German governmental vision Industry 4.0 to support processes connected with the industrial revolution based on CPS: enterprise integration, networking, and digital engineering. The general goals of this initiative are: efficient control of complex distributed systems as a society of autonomous units; integrating the virtual world (where each physical element including sensors, products, human operators, robots and machines is represented by a SW unit/information counterpart) with the physical world (CPS); optimize decision making and efficiency (such as cost effectiveness, high performance and energy efficiency); new business models and approaches to value creation (service orientation, product-service extension, Direct Digital Manufacturing, CMfg ...).

A brief description of the book chapters follows.

Part 1 reports recent advances and on-going research in Cloud and CyberPhysical Systems for Smart Manufacturing. The contributions point at: design of High Availability (HA) CMfg systems integrating distributed MES agents; an analysis framework for the classification of CPS development solutions; formal modelling of distributed automation CPS with CP-agnostic software; integrating advanced data analytics into industrial CPS; the gap analysis on research and innovation for MCPS; development of a redundant and decentralised directory facilitator for resilient Plug and Produce CPS.

Part 2 includes papers devoted to Reconfigurable and Self-organized MultiAgent Systems for Industry and Service. Multi-agent Systems represent the backbone of the distributed intelligent control in manufacturing; however, additional techniques and tools are needed for enhancements: Big Data, ontologies, simulation tools, security modules; predicting the unexpected; fault tolerance and high 
availability. SOA - dual to MAS, is used more and more as implementation of MAS in different layers, providing interoperability between enterprise businesses, MES and shop floor layers. The included papers describe: a self-organising model for mobile robots in large assembly structures using MAS; specification of self-organising logistics systems in terms of openness, intelligence and decentralised control; generic reconfigurable and pluggable material handling system based on genetic algorithm; principles of smart condition-based maintenance for a fleet of mobile entities.

Part 3 introduces Sustainability Issues in Intelligent Manufacturing Systems, analysing the greenness dimension of products, manufacturing processes and systems to maintain equilibrium between economic, social and environmental requirements and constraints. The following subjects are developed: definition of emerging key requirements for future energy-aware production scheduling systems in the MAS and holonic perspective; MAS framework for manufacturing sustainability and optimization; solution for data mining of energy consumption in manufacturing environment; modelling cybersecurity and resilience for softwaredefined networks-based manufacturing applications.

Part 4 includes recent research in the area of Holonic and multi-agent system design for industry and service. Research activities reported in the field focus on holonic and MAS control frameworks that integrate predictive, proactive and reactive mechanisms to provide efficient global batch production performance, and to face increasing unpredicted events. The holonic approach is the main engine for distributing intelligence of MES middle control layer (mixed batch planning and product scheduling, resource allocation, product traceability, production history, predictive maintenance) among agents representing the physical assets, with the scope of rejecting disturbances and reconfiguring shop-floor teams in real-time. In order to cope with both optimality in batch execution and robustness to unforeseen events such as occurrence of rush orders and degradation of resource capabilities, some of the papers included in this section propose holarchies of semi-heterarchical type combining centralized behaviours with decentralized ones, such as the duality System Scheduler-Delegate MAS or the formal specification of a self-sustainable holonic system.

Part 5 groups papers dealing with Dependability and safety of Intelligent Manufacturing Systems. Reliability, availability, security, testability and maintainability of products, processes and systems as well as their greenness are evaluated through dependability and safety studies oriented towards: application of measurement-based AHP to product-driven system control; operational research models and approaches applied to product-driven systems facing unexpected perturbations; holonic facility environment monitoring and control for radiopharmaceutical agent-based production; MAS framework for autonomous real-time resource management of CPS when disruptions are frequent; exploring the design space for myopia-avoiding distributed control systems using classification models.

Part 6 introduces contributions for the Service-oriented Management and Control of Manufacturing Systems. The concepts of services are integrated into holonic manufacturing systems (HMS), leading to Service-oriented HMS. The 
service becomes the main element of negotiation and exchange among holons, by defining:

- How manufacturing services are presented to the system in terms of identification and richness of description (from the manufacturing ontology);

- The strategies and methods to compose complex process workflows by combining individual services to reproduce the desired results;

- The strategies for executing atomic or composite manufacturing services (how to access and invoke such services).

Some papers in this section deal with: dynamic service reconfiguration with MAS tasks; semantic model to perform pluggability of heterogeneous smart devices into the Smart City environment; active monitoring of the product to solve the "lack of information" issue in the use phase.

Part 7 gathers contributions in the field of Engineering and human integration in flexible and reconfigurable industrial systems. These papers analyse how the human operator is integrated in the control architecture of an Intelligent Manufacturing System (IMS). "Human-in-the-loop" concepts consider the intervention of humans (typically for information providing, decision making or direct action on physical components) during the intelligent control of any functions at the operational level of manufacturing, such as scheduling, maintenance, monitoring, supply, etc. The objectives of systems integration evolved in the last few years, shifting from a model where the system was intended to adapt automatically and be equipped with reasoning and decision making capabilities aiming at replacing human ones to a model where CPS are focusing on use scenarios fully involving the humans. With a human-centred design approach in IMS, human resources can be assisted by recently developed ICT tools helping them detecting in advance problems, proposing efficient solutions and taking smart decisions. Representative papers in this section discuss respectively about instantiating the PERFoRM system architecture for industrial case studies, and defining a highly flexible, distributed data analysis framework for Industry 4.0 Manufacturing Systems.

Part 8 is devoted to Virtualization and simulation in computing-oriented industry and service. Papers in this section present resource virtualization techniques and resource sharing in industrial environments. Virtualization of resources and their capabilities allow for manufacturing services encapsulation in the cloud. In CPS (Cyber Physical System) approach of manufacturing, a major challenge is to integrate the computational decisional components (i.e. cyber part) with the physical automation systems and devices (i.e. physical part) to create such network of smart cyber-physical components at MES and shop floor levels. The basic concept of MES and shop floor virtualization involves migration of all workloads that were traditionally executed on physical machines located on the shop floor to the private cloud infrastructure as virtual workloads. The included papers describe: a simulation platform for Virtual Manufacturing Systems (VMS); environments to simulate: distributed agent-based manufacturing systems, adaptable agent-based smart grid 
management and an object-oriented holonic controller for a modular conveyor system.

The studies included in this book show that control paradigms for the manufacturing domain have evolved over time from centralized to decentralized or semi-heterarchical and were mainly driven by the new trends in information and communication technology such as: mobility, connectivity, increase of the decisional capabilities, service orientation and more recently the usage of cloud infrastructures to host control applications, run intensive optimization procedures and store large amount of production and resource data. This is how the concept of cloud manufacturing arose; its scope is to handle manufacturing resources and processes according to the quality of services (QoS) they provide in response to user preferences, and thus efficiently operate and manage them in a unified manner, providing high availability and flexibility in realizing customer orders in both small batches and mass production.

In conventional production structures with multiple workstations which allow job-shop fabrication scenarios, there are several physical control and computing entities that are single points of failure (SPoF) which can be avoided only by hardware redundancy; among these entities which execute centralized tasks are the system scheduler (for high performance computing tasks), the product router (the PLC responsible for routing products throughout the cell towards assigned resources), and the central application for production tracking and resource monitoring.

Private cloud platforms integrated as IaaS in manufacturing solve the high availability problem through resource virtualization techniques and provisioning of computing resources (CPU, storage, I/O) and applications. Also, the system scheduler (SS) implemented in the cloud infrastructure must communicate with the distributed MES (dMES), in which manufacturing resources and product execution orders are agentified; these agents cooperate in a multi-agent framework, both with the SS to receive scheduled orders and report their execution, and between them at dMES level.

Secure communication protocols must be used in such CMfg systems in order to access real-time production data and resource status information from agentified devices: (i) mobile intelligent embedded devices containing both the order execution data (scheduled operations for product execution and assigned resource for each operation) and the WIP data over the product's execution lifecycle; (ii) stationary computing devices providing resource status data and receiving from the SS application programs for assigned operations on products.

The book offers a new integrated vision on Cloud and HPC, Big Data, Analytics and virtualization in Computing-oriented Manufacturing, combining emergent information and communication technologies, service-oriented control of MAS and holonic architectures as well as total enterprise integration solutions based on SOA principles. The MCPS philosophy adopts heterarchical and collaborative control as its information system architecture, based on MAS—SOA duality. In this approach, MAS and Data Analysis are the basic technologies proposed to address the requirements and features envisioned by Industrie 4.0, and also taking in 
consideration the MCPS principles. The first provides the conceptual framework to realize the underline system infrastructure that is required to achieve the desired flexibility and adaptability levels, while the second provides the proper tools capable to analyse and obtain the required information to fulfil the desired system functionalities, also taking advantage of the increased data availability.

These two levels present specific characteristics and requirements which are covered by CPS principles, where the operational level is mainly related with the physical world, characterized by the IoT and its smart devices, also demanding the processing and analysis of real time data streams in order to attend the rapid response monitoring and control requirements. On the other hand, the supervisory level is hosted in a virtual world defined by a cloud-based infrastructure where robust software applications are used to attend the requirements of complex system management and high level information for decision-making, supported by big data analytics.

All these aspects are treated in the present book, which we hope you will find useful reading. 